\title{
Erythropoietin production caused by metastatic colon cancer
}

\author{
Daisuke Ban • Yoshihiro Sakamoto • Kazuaki Shimada • \\ Tomoo Kosuge $\cdot$ Shigeki Sekine $\cdot$ Hirokazu Taniguchi
}

\begin{abstract}
Accepted: 27 August 2009/Published online: 15 September 2009
\end{abstract}
(C) Springer-Verlag 2009

\section{Dear Editor:}

Erythrocytosis is one of the rare paraneoplastic syndromes and can be brought on by ectopic erythropoietin (EPO) production in renal cell carcinomas, cerebral meningiomas, and hepatocellular carcinomas. This syndrome presents with increased hemoglobin levels, pulmonary or deep venous thrombosis, and sometimes requires phlebotomy. We recently treated a patient with metachronous hepatic metastasis from colon cancer, presenting with erythrocytosis, jaundice, and hyperserum EPO concentration. Resection of the tumor normalized the serum EPO concentration and relieved the erythrocytosis. This might be the first case of erythrocytosis secondary to EPO production caused by metastatic colon cancer.

A 56-year-old man had previously undergone resection of a transverse colon cancer in another hospital. Pathological examination revealed adenocarcinoma with subserosal invasion and regional nodal metastases. Before surgery, slight erythrocytosis was found; the patient's hemoglobin level was $17.5 \mathrm{~g} / \mathrm{dl}$ (normal range, 13.8-16.6) and hematocrit was $54.5 \%$ (normal range, 40.2-49.4). One year after colectomy, the hemoglobin level had decreased to $13.5 \mathrm{~g} / \mathrm{dl}$. Adjuvant chemotherapy was performed using 5-fluorouracil and 1leucovorin for 1 year. However, his carcinoembryonic antigen (CEA) level increased to $31.0 \mathrm{ng} / \mathrm{ml}$ and enhanced

D. Ban $\cdot$ Y. Sakamoto $(\bowtie) \cdot K$. Shimada $\cdot$ T. Kosuge

Division of Hepatobiliary and Pancreatic Surgery,

National Cancer Center Hospital,

5-1-1, Tsukiji, Chuo-ku,

Tokyo 104-0045, Japan

e-mail: yosakamo@ncc.go.jp

S. Sekine $\cdot$ H. Taniguchi

Clinical Laboratory, National Cancer Center Hospital,

Tokyo, Japan computed tomography scan showed a heterogeneous, lowdensity tumor, measuring $2.3 \mathrm{~cm}$ in diameter, in the right liver 1.5 year after colectomy. He was referred to our institute for the treatment of the hepatic metastasis. The hemoglobin level and hematocrit had increased to $19.0 \mathrm{~g} / \mathrm{dl}$ and $56.0 \%$, respectively. The patient did not smoke and had no chronic pulmonary or cardiac disease. Bone marrow stem cell culture studies were normal. The serum EPO level was $87.0 \mathrm{mIU} / \mathrm{ml}$ (normal range, 7.4-29.8), and we suspected secondary erythrocytosis caused by the hepatic metastasis because there was no other neoplasm on extensive imaging examinations.

We performed partial resection of the liver, and the serum EPO level decreased $17.0 \mathrm{mIU} / \mathrm{ml}$ on day 7 . The hemoglobin and hematocrit levels returned to their normal range within 1 month. Histopathological findings revealed that the hepatic tumor had metastasized from the primary colon cancer. Immunohistochemical study of the metastatic cells was negative for EPO-antibody. The patient is currently doing well without any recurrence or erythrocytosis 2 years after hepatectomy.

The mechanism of EPO production in cancer cells remains unknown. It is reported that EPO is produced in the fetal liver, but this production is replaced by the kidney soon after birth. Thus, it may be understandable that renal cell carcinoma or hepatocellular carcinoma ectopically produces EPO. On the other hand, production of EPO by adenocarcinoma has not been described in vitro or in vivo. It was obvious that the serum EPO level decreased after resection of the hepatic metastasis, in parallel with the serum CEA level. We suppose that the EPO-producing hepatic metastasis from colon cancer might be extremely rare, although immunohistochemical study of the metastatic cells was negative for EPO-antibody, as a false negative has been reported using monoclonal antibodies. 